\title{
Differential equations for families of semi-classical orthogonal polynomials within class one
}

\author{
G. Filipuk $^{\mathrm{a}, *}$ and M.N. Rebocho ${ }^{\mathrm{b}}$ \\ ${ }^{a}$ Faculty of Mathematics, Informatics and Mechanics, University of Warsaw, \\ Banacha 2, Warsaw, 02-097, Poland; \\ Institute of Mathematics, Polish Academy of Sciences, Śniadeckich 8, Warsaw, \\ 00-656, Poland. \\ ${ }^{\mathrm{b}}$ Departamento de Matemática, Universidade da Beira Interior, 6201-001 Covilhã, \\ Portugal; \\ CMUC, Department of Mathematics, University of Coimbra, 3001-501 Coimbra, \\ Portugal.
}

\begin{abstract}
In this paper we study families of semi-classical orthogonal polynomials of class one. We derive general second or third order ordinary differential equations (with respect to certain parameters) for the recurrence coefficients of the three-term recurrence relation of these polynomials and show that in particular well-known cases, e.g. related to the modified Airy and Laguerre weights, these equations can be reduced to the second and the fourth Painlevé equations.
\end{abstract}

Key words: Painlevé equations, semi-classical orthogonal polynomials, Stieltjes function.

2000 MSC: 34M55, 33C45, 42C05.

\section{Motivation}

The subject matter of the present paper lies within two well-known topics of special functions: semi-classical orthogonal polynomials and the Painlevé equations.

\footnotetext{
* Corresponding author.

Email addresses: filipuk@mimuw.edu.pl (G. Filipuk), mneves@ubi.pt (M.N. Rebocho).
} 
Semi-classical orthogonal polynomials have been extensively studied in the literature, from many points of view $[3,16,18]$. A key feature of semi-classical orthogonal polynomials is the Pearson equation for the weight, $w^{\prime}(z) / w(z)=$ $R(z)$, where $R$ is a rational function of $z$ (see Section 2 for more details). A frequently encountered study in problems in Mathematical Physics is the analysis of modifications of semi-classical weights as functions of some parameters and their consequences for basic structures of the polynomials - the recurrence relation and the deformation derivatives. In this topic, the connections with the Painlevé equations are very well-known, showing that the three-term recurrence relation coefficients are often governed by equations of the Painlevé type (see, for instance, $[2,6,7,8,10,15,16,22]$ ).

Recall that the Painlevé equations $\left(P_{I}\right)-\left(P_{V I}\right)$ are nonlinear second order ordinary differential equations having the property that all movable singularities of an arbitrary solution are at most poles (the so-called Painlevé property). See [9] for various properties, symmetries and application of the Painlevé equations. In this paper we shall deal with the second and the fourth Painlevé equations which are respectively given by

$$
y^{\prime \prime}=2 y^{3}+z y+\alpha \quad P_{I I}(\alpha)
$$

and

$$
y^{\prime \prime}=\frac{y^{\prime 2}}{2 y}+\frac{3 y^{3}}{2}+4 z y^{2}+2\left(z^{2}-\alpha\right) y+\frac{\beta}{y}, \quad P_{I V}(\alpha, \beta)
$$

where $\alpha$ and $\beta$ are arbitrary parameters. Note that by a simple scaling $y(z) \rightarrow$ $i y(-i z)$, where $i^{2}=-1$, equation $P_{I V}(\alpha, \beta)$ is transformed to $P_{I V}(-\alpha, \beta)$. A similar scaling transformation exists also for the second Painlevé equation changing $\alpha$ to $-\alpha$. The second Painlevé equation has classical solutions expressible in terms of the Airy functions if and only if

$$
\alpha=n+1 / 2, \quad n \in \mathbb{Z} .
$$

The fourth Painlevé equation has special solutions expressible in terms of parabolic cylinder functions if and only if either

$$
\beta=-2(2 n+1+\varepsilon \alpha)^{2}, \quad \varepsilon^{2}=1, \quad n \in \mathbb{Z},
$$

or

$$
\beta=-2 n^{2}, \quad n \in \mathbb{Z} .
$$

We shall also need another form of the fourth Painlevé equation, given by

$$
y^{\prime \prime}=\frac{3}{2} \frac{y^{2}}{y}-\beta y^{3}-2\left(z^{2}-\alpha\right) y-4 z-\frac{3}{2 y},
$$

which is obtained from $P_{I V}$ after the change of variables $y(z) \rightarrow 1 / y(z)$. 
In this paper we focus on families of semi-classical orthogonal polynomials in the so-called class one, $w^{\prime} / w=C / A$, under the restrictions $\operatorname{deg}(A) \leq$ $1, \operatorname{deg}(C)=2$, and some of their extensions, through a dependence parameter. The main goal is to obtain second order differential equations (with respect to the parameter) for the three-term recurrence relation coefficients of the orthogonal polynomials. Our approach uses a method similar as in [14]. As examples show, we recover some Painlevé equations in the well-known particular cases. Our results are illustrated by the modified Airy and Laguerre weights from $[7,16,20]$. We show that in these cases our general differential equations are reduced to the second and the fourth Painlevé equations. To the best of our knowledge, we believe that the results in Example 4 are new.

The paper is organised as follows. In Section 2 we present notations as well as results on semi-classical orthogonal polynomials and on discrete Painelvé equations to be used in the sequel. In Section 3 we present the main results of the paper: the derivation of two types of differential equations for the recurrence relation coefficients depending on some parameters to be specified in the text. We illustrate our results by using the well-known modified Airy and Laguerre weights from $[7,16,20]$. Moreover, we obtain conditions under which the general equations are reduced to the second and fourth Painlevé equations up to scaling transformations.

\section{Preliminary results}

\subsection{Semi-classical orthogonal polynomials}

Let $u$ be a linear form defined on the space of polynomials with complex coefficients $\mathbb{P}=\operatorname{span}\left\{x^{k}: k \in \mathbb{N}_{0}\right\}$, and let $\left\{P_{n}(x)=x^{n}+\ldots\right\}$ be the sequence of monic orthogonal polynomials (SMOP) related to $u$, that is,

$$
\left\langle u, P_{n} P_{m}\right\rangle=h_{n} \delta_{n, m}, h_{n} \neq 0, \quad n, m \geq 0 .
$$

It is well known that there exists a sequence of orthogonal polynomials related to $u$ if, and only if, the moments $u_{n}=\left\langle u, x^{n}\right\rangle, n \geq 0$ (where we take $u_{0}=1$ for simplicity), satisfy $\Delta_{n} \neq 0, n \geq 0$, where $\Delta_{n}$ is the Hankel determinant, $\Delta_{n}=\operatorname{det}\left[u_{i+j}\right]_{i, j=0}^{n}, n \geq 0$ (see [21]). Furthermore, if $\Delta_{n}>0, n \geq 0$, then $u$ has an integral representation in terms of a positive Borel measure, $\mu$, supported on an infinite point set, $I$, of the real line

$$
\left\langle u, x^{n}\right\rangle=\int_{I} x^{n} d \mu(x), \quad n \geq 0
$$


and the orthogonality condition (4) becomes

$$
\int_{I} P_{n}(x) P_{m}(x) d \mu(x)=h_{n} \delta_{n, m}, \quad h_{n}>0, \quad n, m \geq 0 .
$$

In the more general case, whenever $\mu$ is an absolutely continuous measure supported on some set $I$, and $w$ denotes its Radon-Nikodym derivative with respect to the Lebesgue measure, i.e. $d \mu(x)=w(x) d x$, then we will also say that $\left\{P_{n}\right\}$ is orthogonal with respect to the weight $w$.

Monic orthogonal polynomials satisfy a three term recurrence relation [21]

$$
P_{n+1}(x)=\left(x-\beta_{n}\right) P_{n}(x)-\gamma_{n} P_{n-1}(x), \quad n \geq 1,
$$

with $P_{0}(x)=1, P_{1}(x)=x-\beta_{0}$, and $\gamma_{n} \neq 0, n \geq 1, \gamma_{0}=1$.

The recurrence relation coefficients, $\gamma_{n}$ and $\beta_{n}$, are given by

$$
\gamma_{0}=1, \quad \gamma_{n+1}=\frac{\left\langle u, x P_{n} P_{n-1}\right\rangle}{\left\langle u, P_{n-1}^{2}\right\rangle}, \quad \beta_{n}=\frac{\left\langle u, x P_{n}^{2}\right\rangle}{\left\langle u, P_{n}^{2}\right\rangle}, \quad n \geq 0
$$

We also remark that the recurrence relation coefficients can also be computed by using the ratios of Hankel determinants [21],

$$
\gamma_{n+1}=\frac{\Delta_{n-1} \Delta_{n+1}}{\Delta_{n}^{2}}, \Delta_{-1}=1
$$

The constant $h_{n}$ in (4) is given by

$$
h_{0}=1, \quad h_{n}=\prod_{k=1}^{n} \gamma_{k}, \quad n \geq 1
$$

We introduce the moment generating function, the Stieltjes function, defined by $S(x)=\sum_{n=0}^{+\infty} u_{n} x^{-n-1}$. Note that whenever $u$ is defined by a measure such as (5), then $S$ is given by

$$
S(x)=\int_{I} \frac{d \mu(s)}{x-s}, \quad x \in \mathbb{C} \backslash I .
$$

The sequence of functions of the second kind corresponding to $\left\{P_{n}\right\}$ is defined by

$$
q_{n+1}=P_{n+1} S-P_{n}^{(1)}, \quad n \geq 0, q_{0}=S,
$$

where $P_{n}^{(1)}$ is the so-called associated polynomial of $P_{n}$ of degree $n$ [21]. Whenever we are dealing with measures such as in (5), then there holds the integral 
representation

$$
q_{n+1}(x)=\int_{I} \frac{P_{n+1}(s)}{x-s} d \mu(s), \quad n \geq 0, q_{0}=S .
$$

Note that $\left\{q_{n}\right\}$ satisfies the recurrence relation (6), with initial conditions $q_{-1}=0, q_{0}(x)=S(x)$. Thus, the following asymptotic expansions hold (see, e.g., $[20])$ :

$$
\begin{aligned}
& P_{n}(x)=x^{n}-\left(\sum_{j=0}^{n-1} \beta_{j}\right) x^{n-1}+\left(\sum_{j=1}^{n-1}\left(\sum_{i=0}^{j-1} \beta_{i} \beta_{j}-\gamma_{j}\right)\right) x^{n-2}+\ldots, \\
& q_{n}(x)=h_{n}\left(\frac{1}{x^{n+1}}+\left(\sum_{j=0}^{n} \beta_{j}\right) \frac{1}{x^{n+2}}+\sum_{j=0}^{n}\left(\gamma_{j+1}+\sum_{i=0}^{j} \beta_{i} \beta_{j}\right) \frac{1}{x^{n+3}}+\ldots \text { (1) } 1\right)
\end{aligned}
$$

Also, recall the following equality, which can be obtained from the ChristoffelDarboux identity $[5,21]$

$$
P_{n+1} q_{n}-P_{n} q_{n+1}=h_{n}, \quad n \geq 0 .
$$

Semi-classical orthogonal polynomials are defined as sequences of orthogonal polynomials related to linear functionals $u$ that satisfy a distributional equation with polynomial coefficients [18],

$$
\mathcal{D}(A u)=\psi u,
$$

being $\mathcal{D}$ the derivative operator. To Eq. (13) one associates the class [18], a non-negative integer given by the minimum value of $\max \{\operatorname{deg}(\psi)-1, \operatorname{deg}(A)-$ $2\}$, for all pairs of polynomials $(A, \psi)$ satisfying (13).

Equation (13) is equivalent to a differential equation for the Stieltjes function,

$$
A S^{\prime}=C S+D, C=\psi-A^{\prime},
$$

with $D$ a polynomial given in terms of $A, C$.

If $u$ is positive-definite admitting an integral representation via a weight function $w$ with support on the real line, then the semi-classical character of $u$ is equivalent to the so-called Pearson equation

$$
A w^{\prime}=C w
$$

Here, $C$ is the same an in (14). 
In the present paper we shall consider the class one. Essentially, we will consider weight functions $w$ satisfying (15) under conditions

$$
\operatorname{deg}(A) \leq 3, \operatorname{deg}(C)=2 \text { or } \operatorname{deg}(A)=3, \operatorname{deg}(C)<2 .
$$

In the sequel we will use the following matrices, for $n \geq 0$ :

$$
\mathcal{Y}_{n}=\left[\begin{array}{cc}
P_{n+1} & q_{n+1} / w \\
P_{n} & q_{n} / w
\end{array}\right]
$$

which satisfy the difference equation

$$
\mathcal{Y}_{n}=\mathcal{A}_{n} \mathcal{Y}_{n-1}, \quad \mathcal{A}_{n}=\left[\begin{array}{cc}
x-\beta_{n}-\gamma_{n} \\
1 & 0
\end{array}\right], \quad n \geq 1
$$

with initial conditions $\mathcal{Y}_{0}=\left[\begin{array}{cc}x-\beta_{0} & q_{1} / w \\ 1 & q_{0} / w\end{array}\right]$.

The theorem that follows gives a characterization of semi-classical weights.

Theorem $1[4,16]$ Let $\left\{P_{n}\right\}$ be a SMOP with respect to a weight $w$, and let $\left\{q_{n}\right\}$ be the corresponding sequence of functions of the second kind. The weight $w$ is semi-classical and satisfies $w^{\prime} / w=C / A$ if, and only if, $\mathcal{Y}_{n}=$ $\left[\begin{array}{cc}P_{n+1} & q_{n+1} / w \\ P_{n} & q_{n} / w\end{array}\right]$ satisfies the differential system

$$
A \mathcal{Y}_{n}^{\prime}=\left(\mathcal{B}_{n}-C / 2 I\right) \mathcal{Y}_{n}, \quad n \geq 1,
$$

where $I$ is the identity matrix and

$$
\mathcal{B}_{n}=\left[\begin{array}{cc}
l_{n} & \Theta_{n} \\
-\Theta_{n-1} / \gamma_{n} & -l_{n}
\end{array}\right]
$$

with $l_{n}, \Theta_{n}$ being polynomials of uniformly bounded degrees.

Remark 1 Let us take $w^{\prime} / w=C / A$ under conditions (16). Set

$$
\begin{gathered}
A(x)=a_{3} x^{3}+a_{2} x^{2}+a_{1} x+a_{0}, \quad C(x)=c_{2} x^{2}+c_{1} x+c_{0}, \\
l_{n}(x)=\ell_{n, 2} x^{2}+\ell_{n, 1} x+\ell_{n, 0}, \quad \Theta_{n}(x)=\theta_{n, 1} x+\theta_{n, 0} .
\end{gathered}
$$

By equating coefficients in (18), we have 


$$
\begin{aligned}
& \ell_{n, 2}=(n+1) a_{3}+c_{2} / 2 \\
& \ell_{n, 1}=a_{3}\left(\eta_{n}+\beta_{0}\right)+(n+1) a_{2}+c_{1} / 2 \\
& \ell_{n, 0}=\lambda_{n, 0}-\theta_{n, 1} \\
& \lambda_{n, 0}=-2 a_{3}\left(\nu_{n}+\beta_{0} \eta_{n}-\gamma_{1}\right)+(n+1) a_{1}+c_{0} / 2 \\
& \quad+\left(\eta_{n}+\beta_{0}\right)\left(a_{3}\left(\eta_{n}+\beta_{0}\right)+a_{2}\right) \\
& \theta_{n, 1}=-\gamma_{n+1}\left((2 n+3) a_{3}+c_{2}\right), \quad \\
& \theta_{n, 0}=-\gamma_{n+1}\left\{2 a_{3}\left(\eta_{n}+(n+2) \beta_{n+1}+\beta_{0}\right)+a_{2}(2 n+3)+c_{2} \beta_{n+1}+c_{1}\right\}
\end{aligned}
$$

with

Also, we have

$$
\eta_{n}=\sum_{k=1}^{n} \beta_{k}, \quad \nu_{n}=\sum_{1 \leq i<j \leq n}^{n} \beta_{i} \beta_{j}-\sum_{k=2}^{n} \gamma_{k}, \quad n \geq 1 .
$$

$$
a_{1}+c_{0}+\left(2 a_{2}+c_{1}\right) \beta_{0}+\left(3 a_{3}+c_{2}\right) \beta_{0}^{2}+\left(3 a_{3}+c_{2}\right) \gamma_{1}=0 .
$$

Condition (27) can also be obtained by finding the expansion of the Stieltjes function $S$ at infinity from the differential equation $A S^{\prime}=C S+D$. We can find the first few moments $u_{0}=1, u_{1}=\beta_{0}, u_{2}=-\left(1+c_{0}+c_{1} \beta_{0}\right) / c_{2}$ and by computing $\gamma_{1}$ using (7) we get (27).

Throughout the paper, $f^{\prime}$ will denote the derivative of $f$ with respect to $x$ and $\dot{f}$ denotes the derivative of $f$ with respect to some parameter to be specified in the context.

2.2 Discrete Painlevé equations for the recurrence relation coefficients of semi-classical orthogonal polynomials

We collect some results on discrete Painlevé equations for semi-classical orthogonal polynomials of class one. They follow from [13], taking $B \equiv 0$. These results concern differential equations for the Stieltjes function, $A S^{\prime}=C S+D$ under the assumption $\operatorname{deg}(A) \leq 1$.

Theorem 2 [13] Let $S$ be a Stieltjes function satisfying $A S^{\prime}=C S+D$ with

$$
A(x)=a_{0} \neq 0, C(x)=c_{2} x^{2}+c_{1} x+c_{0}, D(x)=d_{1} x+d_{0},
$$

where $d_{1}=-c_{2}, d_{0}=-c_{2} \beta_{0}-c_{1}$ and $c_{2} \neq 0$. Let $\left\{P_{n}\right\}$ be the SMOP associated with $S$, satisfying the recurrence relation (6),

$$
P_{n+1}(x)=\left(x-\beta_{n}\right) P_{n}(x)-\gamma_{n} P_{n-1}(x), \quad n=0,1,2, \ldots,
$$

with $\beta_{n} \neq 0, \quad n=0,1,2, \ldots$

Set $\eta=c_{2}, \mu=c_{0} / 2, \lambda=c_{1}$. The recurrence relation coefficients $\beta_{n}, \gamma_{n}$ are related through the following discrete system: 


$$
\begin{aligned}
& \beta_{n}\left(\eta \beta_{n}+\lambda\right)=-\left(\eta \gamma_{n}+\mu\right)-\left(\eta \gamma_{n+1}+\mu\right), \quad n \geq 1, \\
& \eta\left(\beta_{n}+\beta_{n+1}\right)+\lambda=\frac{-n a_{0}+\gamma_{1}\left(\eta\left(\beta_{0}+\beta_{1}\right)+\lambda\right)}{\gamma_{n+1}}, \quad n \geq 1 .
\end{aligned}
$$

Moreover, the sequences

$$
x_{n}=\eta \beta_{n}+\lambda / 2, \quad z_{n}=\eta^{2}\left(-n a_{0}+\gamma_{1}\left(\eta\left(\beta_{0}+\beta_{1}\right)+\lambda\right)\right)
$$

satisfy the alternative discrete Painlevé equation $d P_{I}$

$$
\frac{z_{n-1}}{x_{n-1}+x_{n}}+\frac{z_{n}}{x_{n}+x_{n+1}}=-x_{n}^{2}+\gamma, \quad \gamma=(\lambda / 2)^{2}-2 \mu \eta
$$

with the initial conditions $x_{0}=\eta \beta_{0}+\lambda / 2, \quad x_{1}=\eta \beta_{1}+\lambda / 2$.

Theorem 3 [13] Let $S$ be a Stieltjes function satisfying $A S^{\prime}=C S+D$ with

$$
A(x)=x-t, C(x)=c_{2} x^{2}+c_{1} x+c_{0}, D(x)=d_{1} x+d_{0},
$$

where $d_{1}=-c_{2}, d_{0}=-c_{2} \beta_{0}-c_{1}$ and $c_{2} \neq 0$. Let $\left\{P_{n}\right\}$ be the SMOP associated with $S$, satisfying the recurrence relation (6),

$$
P_{n+1}(x)=\left(x-\beta_{n}\right) P_{n}(x)-\gamma_{n} P_{n-1}(x), \quad n=0,1,2, \ldots
$$

Then the sequences

$$
\begin{gathered}
x_{n}=\frac{k_{1} c_{2}}{k_{2}\left(c_{1}+c_{2} t\right)+\beta_{n} c_{2}}, \\
y_{n}=\frac{k_{2}^{2}\left(2 n+c_{0}+2 c_{2} \gamma_{n}+c_{2} t^{2}+c_{1} t\right)}{2 c_{2} k_{1}^{2}}
\end{gathered}
$$

satisfy

$$
x_{n-1} x_{n}=\frac{2\left(k_{2}^{2}\left(2 n+c_{0}+c_{2} t^{2}+c_{1} t\right)-2 c_{2} k_{1}^{2} y_{n}\right)}{c_{2} k_{1}^{2}\left(c_{0}^{2}-4 y_{n}^{2}\right)}
$$

and

$$
y_{n}+y_{n+1}=\frac{k_{2} x_{n}\left(c_{1}+2 c_{2} t\right)-c_{2} k_{1}}{c_{2} k_{1} x_{n}^{2}},
$$

where $k_{1}$ and $k_{2}$ are constants. The initial conditions are given by

$$
x_{0}=\frac{c_{2} k_{1}}{k_{2}\left(c_{1}+c_{2} \beta_{0}+c_{2} t\right)}
$$

and $y_{0}$, which is obtained from the formulae above with $n=0$. 
2.3 Differential Toda-type systems for the recurrence relation coefficients of semi-classical orthogonal polynomials

There might be parameters in the weight, and so the recurrence relation coefficients become functions of these parameters. In case the weight is of the form $w(x) \exp (t x)$, the coefficients of the orthogonal polynomials depend on $t$ and they satisfy the Toda system (this is a well-known result, see, for instance, $[14,19]$ and the references therein).

Theorem 4 [19] The recurrence relation coefficients of polynomials orthogonal with respect to the weight $w=w_{0}(x) \exp (t x)$ on the real line satisfy the Toda system

$$
\begin{aligned}
& \dot{\gamma}_{n}=\gamma_{n}\left(\beta_{n}-\beta_{n-1}\right), \\
& \dot{\beta}_{n}=\gamma_{n+1}-\gamma_{n} .
\end{aligned}
$$

The initial conditions $\beta_{n}(0)$ and $\gamma_{n}(0)$ correspond to the recurrence relation coefficients of the orthogonal polynomials for the weight $w_{0}(x)$.

However, the weight might depend on the parameter in a different way. The result that follows is given in [16, Theorem 2], but here we state it according to our notation for SMOP.

Theorem 5 [16] Let $\left\{P_{n}\right\}$ be the SMOP with respect to the semi-classical weight $w$ satisfying $w^{\prime} / w=C / A$ with the conditions

(i) $A(x)=\prod_{k=1}^{m}\left(x-x_{k}\right), x_{i} \neq x_{j}, i \neq j$

(ii) the residues $\epsilon_{k}=C\left(x_{k}\right) / A^{\prime}\left(x_{k}\right)$ are not integers, $k=1, \ldots, m$,

and where we assume that at least one of the $x_{k}$ 's depend on a parameter $t$. Then, we have the following Toda-type equations:

$$
\begin{aligned}
& \frac{\dot{\gamma_{n}}}{\gamma_{n}}=\sum_{k=1}^{m}\left(\frac{\Theta_{n-2}\left(x_{k}\right)}{\gamma_{n-1}}-\frac{\Theta_{n-1}\left(x_{k}\right)}{\gamma_{n}}\right) \frac{\dot{x}_{k}}{A^{\prime}\left(x_{k}\right)}, \\
& \dot{\beta}_{n}=\sum_{k=1}^{m}\left(l_{n}\left(x_{k}\right)-l_{n-1}\left(x_{k}\right)\right) \frac{\dot{x}_{k}}{A^{\prime}\left(x_{k}\right)},
\end{aligned}
$$

where $l_{n}, \Theta_{n}$ are the polynomials introduced in Theorem 1.

Next we give some remarks on the proof of Theorem 5 which will be used later on. 
Remark 2 Under the notations of Theorem 1, define the matrix $\mathcal{H}_{n}$ such that $\dot{\mathcal{Y}}_{n}=\mathcal{H}_{n} \mathcal{Y}_{n}$, that is,

$$
\mathcal{H}_{n}=\dot{\mathcal{Y}}_{n} \mathcal{Y}_{n}^{-1}
$$

Thus, we have

$\mathcal{H}_{n}=\frac{1}{h_{n}}\left[\begin{array}{cc}\dot{P}_{n+1} q_{n}-P_{n} \dot{q}_{n+1}+\frac{\dot{w}}{w} q_{n+1} P_{n} & -\dot{P}_{n+1} q_{n+1}+P_{n+1} \dot{q}_{n+1}-\frac{\dot{w}}{w} q_{n+1} P_{n+1} \\ \dot{P}_{n} q_{n}-P_{n} \dot{q}_{n}+\frac{\dot{w}}{w} q_{n} P_{n} & -\dot{P}_{n} q_{n+1}+P_{n+1} \dot{q}_{n}-\frac{\dot{w}}{w} q_{n} P_{n+1}\end{array}\right]$

with $h_{n}$ given in (8), and where (12) was used to compute $\operatorname{det}\left(\mathcal{Y}_{n}\right)$.

Recall the system given in (18), $A \mathcal{Y}_{n}^{\prime}=\left(\mathcal{B}_{n}-C / 2 I\right) \mathcal{Y}_{n}$. The compatibility between $\frac{\partial \mathcal{Y}_{n}^{\prime}}{\partial t}$ and $\frac{\partial \dot{\mathcal{Y}}_{n}}{\partial z}$ yields

$$
\frac{\partial}{\partial t}\left(\frac{\mathcal{B}_{n}-C / 2 I}{A}\right)=\mathcal{H}_{n}^{\prime}+\mathcal{H}_{n} \frac{\mathcal{B}_{n}}{A}-\frac{\mathcal{B}_{n}}{A} \mathcal{H}_{n}
$$

Then, equations (38)-(39) follow from (42), taking into account the asymptotic expansion of (41) (for details see [16, pp. 224-225]).

Furthermore, it should be emphasized that equation (42) is of particular relevance when dealing with the case $\operatorname{deg}(A)=0$. In such a case, assuming that $\dot{w} / w=E / A$, with $E$ some polynomial, the matrix $\mathcal{H}_{n}$ is now expected to have polynomial entries (see [12, Section 2]). Hence, for some degrees of $A, C, E$, the matrices $\mathcal{B}_{n}, \mathcal{H}_{n}$ are easy to determine, and, consequently, differential equations for the recurrence relation coefficients $\beta_{n}, \gamma_{n}$ are expected to be enclosed.

\section{Main results}

\subsection{Case $\operatorname{deg}(A)=0$}

In this subsection we shall deal with the weight $w$ that satisfies the differential equation of the form $w^{\prime} / w=\left(c_{2} x^{2}+c_{1} x+c_{0}\right) / a_{0}$. Let us write $w^{\prime} / w=$ $\tilde{c}_{2} x^{2}+\tilde{c}_{1} x+\tilde{c}_{0}$. The general solution is given by the exponential cubic weight,

$$
w(x)=c \exp \left(\tilde{c}_{0} x+\tilde{c}_{1} x^{2} / 2+\tilde{c}_{2} x^{3} / 3\right),
$$

where $c$ is an arbitrary constant. 


\subsubsection{Differential equations using Toda systems}

In this subsection we assume the dependence on the parameter of the form $w=w_{0}(x) \exp (t x)$. Then, the coefficients of the Pearson equation for $w$ depend on $t$. Note that the recurrence relation coefficients, $\beta_{n}=\beta_{n}(t)$ and $\gamma_{n}=\gamma_{n}(t)$, satisfy the discrete equations (28), (29) as well as the differential equations (36), (37).

The main objective of this subsection is to deduce a differential equation for $\beta_{n}$ and to show how in the particular case of the modified Airy weight such an equation is reduced to the second Painlevé equation.

Theorem 6 Let $w$ be a semi-classical weight such that $w^{\prime} / w=C / A$, with

$$
A(x)=a_{0} \neq 0, C(x)=c_{2} x^{2}+c_{1} x+c_{0}, \quad c_{2} \neq 0 .
$$

Let $\left\{P_{n}\right\}$ be the SMOP with respect to $w$, satisfying the recurrence relation (6),

$$
P_{n+1}(x)=\left(x-\beta_{n}\right) P_{n}(x)-\gamma_{n} P_{n-1}(x), \quad n=0,1,2, \ldots,
$$

with $\beta_{n} \neq 0, \quad n=0,1,2, \ldots$

Set $\eta=c_{2}, \mu=c_{0} / 2, \lambda=c_{1}$. Assuming a $t$-dependence in the weight of the form $w_{0}(x) \exp (t x)$, the recurrence relation coefficients $\beta_{n}=\beta_{n}(t), n \geq 1$, satisfy the following second order differential equation

$$
\eta^{2} \ddot{\beta}_{n}=2 \eta^{2} \beta_{n}^{3}+3 \eta \lambda \beta_{n}^{2}+H_{1} \beta_{n}+H_{2},
$$

where

$H_{1}=\lambda^{2}+4 \eta \mu-\lambda \dot{\eta}+\eta \dot{\lambda}, H_{2}=2 \gamma_{1} \eta\left(\lambda+\eta\left(\beta_{0}+\beta_{1}\right)\right)+2 \lambda \mu+2 \eta \dot{\mu}-2 \mu \dot{\eta}-2 n a_{0} \eta$.

Here we assume that $a_{0}, \eta, \lambda$ and $\mu$ depend on $t$.

PROOF. To obtain the differential equation (43), we first take the four equations (28), (29), (36) with $n$ replaced by $n+1$, and (37). From the first equation we find $\gamma_{n}$. From the second equation we find $\beta_{n+1}$. Next we substitute these expressions into the third and the fourth equations. Finally, eliminating $\gamma_{n+1}$ between the resulting equations, we get a second order differential equation for $\beta_{n}$.

Note that using (27) and (36) with $n=1$ we can express $\beta_{1}$ in terms of $\dot{\beta}_{0}$ and $\beta_{0}$ (see example below).

Theorem 7 Under assumptions in Theorem 6 , the recurrence relation coefficient $\beta_{n}=\beta_{n}(t), n \geq 1$, satisfies the following third order differential 
equation:

$$
\begin{gathered}
\eta^{3} \dddot{\beta}_{n}= \\
+\left(6 \eta^{2} \beta_{n}^{2}+6 \eta \lambda \beta_{n}+\lambda^{2}+4 \eta \mu-2 \lambda \dot{\eta}+2 \eta \dot{\lambda}\right) \dot{\beta}_{n}+\eta(2 \eta \dot{\lambda}-2 \lambda \dot{\eta}) \beta_{n}^{2} \\
+\left(\eta^{2} \ddot{\lambda}-\eta \lambda \ddot{\eta}+4 \eta^{2} \dot{\mu}-2 \eta \dot{\eta} \dot{\lambda}+\eta \lambda \dot{\lambda}+2 \lambda \dot{\eta}^{2}-4 \eta \mu \dot{\eta}-\lambda^{2} \dot{\eta}\right) \beta_{n} \\
-2 \lambda \mu \dot{\eta}+4 \mu \dot{\eta}^{2}+2 \eta \lambda \dot{\mu}-4 \eta \dot{\eta} \dot{\mu}-2 \eta \mu \ddot{\eta}+3 \eta^{2} \ddot{\mu}
\end{gathered}
$$

PROOF. Take equations (28), (36) with $n$ replaced by $n+1$, and (37). From the first equation we get $\gamma_{n+1}$. Substituting this expression into the other two equations, we obtain two equations: the first one involves $\dot{\gamma}_{n}, \gamma_{n}, \beta_{n}, \beta_{n+1}$, and the second one involves $\dot{\beta}_{n}, \beta_{n}, \gamma_{n}$. Augmenting these two equations with the last with $n$ replaced by $n+1$, we get a system of three equations from where we eliminate $\beta_{n+1}$ and $\gamma_{n}$, thus obtaining a third order equation for $\beta_{n}$.

Example 1 Take $w(x)=\exp \left(x^{3} / 3+t x\right)$, the modified Airy weight with the appropriate contour of integration in the complex plane. It is known (see [16] and the references therein) that the recurrence relation coefficients are related to the second Painleve equation. Let us show how to reproduce this result using (43). Here $A(x)=1, C(x)=x^{2}+t$. Equation (43) becomes

$$
\ddot{\beta}_{n}=2 \beta_{n}^{3}+2 t \beta_{n}+2 \gamma_{1}\left(\beta_{0}+\beta_{1}\right)-2 n+1 .
$$

Condition (27) is then $t+\beta_{0}^{2}+\gamma_{1}=0$. Differentiating this equation with respect to $t$ and using equation $\dot{\gamma}_{1}=\gamma_{1}\left(\beta_{1}-\beta_{0}\right)=-\left(t+\beta_{0}^{2}\right)\left(\beta_{1}-\beta_{0}\right)$ from (36) with $n=1$, we get an expression for $\beta_{0}, \dot{\beta}_{0}, \beta_{1}$, from which we can find $\beta_{1}$. Explicitly,

$$
\beta_{1}=\frac{1+t \beta_{0}+\beta_{0}^{3}+2 \beta_{0} \dot{\beta}_{0}}{t+\beta_{0}^{2}} .
$$

We can substitute this formula into (45) and get

$$
\ddot{\beta}_{n}=2 \beta_{n}^{3}+2 t \beta_{n}-4 \beta_{0}^{3}-4 \beta_{0}\left(t+\dot{\beta}_{0}\right)-2 n-1 \text {. }
$$

If

$$
\dot{\beta}_{0}=-t-\beta_{0}^{2},
$$

then we get the second Painlevé equation (up to scaling of variables) for the function $\beta_{n}$, namely

$$
\ddot{\beta}_{n}=2 \beta_{n}^{3}+2 t \beta_{n}-2 n-1 .
$$

By changing variables $\beta_{n}(t) \rightarrow 2^{1 / 3} y\left(2^{1 / 3} t\right)$ we get $P_{I I}(-n+1 / 2)$ for $y(z)$, where $z=2^{1 / 3} t$. Note that parameters of $P_{I I}$ in this case are as in (1). A standard linearization of (46) gives the Airy equation. Moreover, when taking $n=0$ in equation (47), we can easily show that all solutions of (46) satisfy it. Thus, we get a family of classical solutions of the second Painleve equation which are expressible in terms of the Airy function for the function $\beta_{0}$. 
Equation (7) is reduced to

$$
\dddot{\beta}_{n}=6 \beta_{n}^{2} \dot{\beta}_{n}+2 t \dot{\beta}_{n}+2 \beta_{n}
$$

and, clearly, (47) solves it.

Moreover, we see that in order to reduce equation (43) to the second Painlevé equation up to scaling transformations, we need conditions on the coefficients of $C(x)$ (for instance, if $c_{2}=1$, then $c_{1}=0$ and $c_{0}$ is linear), which gives only very special weights.

\subsubsection{Differential equations using Toda-type systems}

We can also derive similar equations with respect to some parameter $t$. Indeed, not necessarily when $w=w_{0}(x) \exp (t x)$.

Lemma 1 Let $w$ be a semi-classical weight under some dependence on a parameter $t$, satisfying $w^{\prime} / w=C / A, \dot{w} / w=E / A$, with $A(x)=a_{0}, C(x)=$ $c_{2} x^{2}+c_{1} x+c_{0}, E(x)=e_{2} x^{2}+e_{1} x+e_{0}$. Let $\left\{P_{n}\right\}$ be the SMOP with respect to $w$, satisfying the recurrence relation (6),

$$
P_{n+1}(x)=\left(x-\beta_{n}\right) P_{n}(x)-\gamma_{n} P_{n-1}(x), \quad n=0,1,2, \ldots .
$$

The matrix $\mathcal{H}_{n}$ defined in (41) is given as follows:

$$
\mathcal{H}_{n}=\left[\begin{array}{cc}
\gamma_{n+1} \frac{e_{2}}{a_{0}} & -\gamma_{n+1}\left(\frac{e_{2}}{a_{0}}\left(x+\beta_{n+1}\right)+\frac{e_{1}}{a_{0}}\right) \\
\frac{e_{2}}{a_{0}}\left(x+\beta_{n}\right)+\frac{e_{1}}{a_{0}} & \frac{\dot{h}_{n}}{h_{n}}-\frac{e_{2}}{a_{0}}\left(x^{2}+\gamma_{n+1}+\lambda_{n}\right)-\frac{e_{1}}{a_{0}} x-\frac{e_{0}}{a_{0}}
\end{array}\right]
$$

where

$$
\lambda_{n}=\sum_{j=0}^{n}\left(\sum_{i=0}^{j} \beta_{i} \beta_{j}\right)+\sum_{j=1}^{n}\left(\sum_{i=0}^{j-1} \beta_{i} \beta_{j}\right)-\left(\sum_{j=0}^{n} \beta_{j}\right)^{2} .
$$

PROOF. Under the assumption $\dot{w} / w=E / A$ with $E$ a polynomial, the matrix $\mathcal{H}_{n}$ has polynomial entries (see [12, Section 2]). Thus, in the account of (41) and the asymptotic expansions (10) and (11), the terms in $\mathcal{O}\left(x^{k}\right), k \geq 0$, are obtained from

$$
\frac{1}{h_{n}}\left[\begin{array}{cc}
\frac{\dot{w}}{w} q_{n+1} P_{n} & -\frac{\dot{w}}{w} q_{n+1} P_{n+1} \\
\frac{\dot{w}}{w} q_{n} P_{n} & P_{n+1} \dot{q}_{n}-\frac{\dot{w}}{w} q_{n} P_{n+1}
\end{array}\right] .
$$

The use of the asymptotic expansions (10) and (11) in (49), together with $\gamma_{n+1}=h_{n+1} / h_{n}$ (cf. (8)), yields (48). 
Theorem 8 Under the conditions and notations in Lemma 1, equation (42) gives the following Toda-type system for the recurrence relation coefficients:

$$
\begin{gathered}
a_{0} c_{2} \dot{\gamma}_{n+1}=\left(e_{2} c_{1}-e_{1} c_{2}\right)\left(\beta_{n}-\beta_{n+1}\right) \gamma_{n+1}, \\
a_{0} c_{2}^{2} \dot{\beta}_{n}=e_{1} c_{2}\left(c_{0}+c_{1} \beta_{n}+c_{2}\left(\beta_{n}^{2}+2 \gamma_{n+1}\right)\right) \\
-e_{2}\left(a_{0} c_{2}+c_{1}\left(c_{0}+c_{1} \beta_{n}+c_{2}\left(\beta_{n}^{2}+2 \gamma_{n+1}\right)\right)\right), \\
\left(e_{2} c_{1}-e_{1} c_{2}\right)\left(c_{0}+c_{1} \beta_{n+1}+c_{2}\left(\beta_{n+1}^{2}+\gamma_{n+1}+\gamma_{n+2}\right)\right)=0, \\
c_{0} \dot{a}_{0}+a_{0}\left(e_{1}-\dot{c}_{0}\right)=0, \quad 2 a_{0} e_{2}+c_{1} \dot{a}_{0}-a_{0} \dot{c}_{1}=0, \quad c_{2} \dot{a}_{0}-a_{0} \dot{c}_{2}=0 .
\end{gathered}
$$

The second factor in condition (52) is equation (28) with $n$ replaced by $n+1$. Moreover, system (50), (8), (52) implies

$$
\left(e_{2} c_{1}-e_{1} c_{2}\right)\left(\beta_{n}-\beta_{n+1}\right)\left(c_{1}+c_{2}\left(\beta_{n}+\beta_{n+1}\right)\right)+a_{0} c_{2}\left(2 e_{2}+c_{2}\left(\dot{\beta}_{n}+\dot{\beta}_{n+1}\right)\right)=0 .
$$

PROOF. The entries of the matrix $\mathcal{B}_{n}$ defined in (19) are given by

$$
l_{n}(x)=\frac{c_{2}}{2} x^{2}+\frac{c_{1}}{2} x+\frac{c_{0}}{2}+c_{2} \gamma_{n+1}, \Theta_{n}(x)=-\gamma_{n+1}\left(c_{2} x+c_{2} \beta_{n+1}+c_{1}\right) .
$$

By collecting the coefficients in $x$ in (42) and eliminating $\dot{h}_{n}$, we obtain the result after simplifying expressions using (53).

Example 2 Let us take $w(x)=\exp \left(x^{3} / 3+t x\right)$. This example was already studied in [16]. However, we shall use equations derived in this subsection.

We have $A(x)=1, C(x)=x^{2}+t, E(x)=x$, and

$$
\mathcal{B}_{n}=\left[\begin{array}{cc}
\frac{x^{2}}{2}+\frac{t}{2}+\gamma_{n+1} & -\gamma_{n+1}\left(x+\beta_{n+1}\right) \\
x+\beta_{n} & -\left(\frac{x^{2}}{2}+\frac{t}{2}+\gamma_{n+1}\right)
\end{array}\right], \quad \mathcal{H}_{n}=\left[\begin{array}{cc}
0 & -\gamma_{n+1} \\
1 & -x+\frac{\dot{h}_{n}}{h_{n}}
\end{array}\right]
$$

Theorem 8 gives

$$
\begin{gathered}
\gamma_{n+1}=\left(\beta_{n+1}-\beta_{n}\right) \gamma_{n+1}, \\
\dot{\beta}_{n}=t+2 \gamma_{n+1}+\beta_{n}^{2}, \\
\gamma_{n+2}=-\gamma_{n+1}-\beta_{n+1}^{2}-t, \\
\dot{\beta}_{n+1}+\dot{\beta}_{n}=\beta_{n}^{2}-\beta_{n+1}^{2} .
\end{gathered}
$$

Note that equation (55) is the same as equation (36). By simple manipulations we can also recover (37). The second and the third order differential equations for $\beta_{n}$ are the same as in the previous subsection. 


\subsection{Case $\operatorname{deg}(A)=1$}

In this subsection we shall deal with the weight $w$ that satisfies the differential equation of the form $w^{\prime} / w=\left(c_{2} x^{2}+c_{1} x+c_{0}\right) /(x-\alpha)$ with the general solution

$$
w(x)=c e^{\frac{1}{2}(x-\alpha)\left(c_{2}(3 \alpha+x)+2 c_{1}\right)}(x-\alpha)^{\alpha\left(\alpha c_{2}+c_{1}\right)+c_{0}},
$$

where $c$ is an arbitrary constant.

In the subsections below we will use the same method as in [14] to derive differential equations. It is as follows.

Assume that we are given six equations of the form

$$
\begin{aligned}
& f\left(x_{n}, \beta_{n}\right)=0, \\
& g\left(y_{n}, \gamma_{n}\right)=0, \\
& f_{1}\left(x_{n-1}, x_{n}, y_{n}\right)=0, \\
& f_{2}\left(x_{n}, y_{n}, y_{n+1}\right)=0, \\
& g_{1}\left(\beta_{n-1}, \beta_{n}, \gamma_{n}, \dot{\gamma}_{n}\right)=0, \\
& g_{2}\left(\dot{\beta}_{n}, \gamma_{n}, \gamma_{n+1}\right)=0,
\end{aligned}
$$

where the functions on the left-hand sides also depend on the coefficients (as functions of some parameter $t$ ) of the Pearson equation for the weight, and $x_{n}=x_{n}(t), y_{n}=y_{n}(t), \beta_{n}=\beta_{n}(t), \gamma_{n}=\gamma_{n}(t)$. Equations (59), (60) introduce new quantities $x_{n}$ and $y_{n}$ related to the recurrence relation coefficients; equations (61), (62) are discrete equations as in Theorem 3; equations (63), (64) are the Toda-type equations. The aim is to derive an ordinary differential equation for the function $x_{n}$. To do this we first find expression of $x_{n-1}$ in terms of $x_{n}$ and $y_{n}$ to be used later on from equation (61). From equations (59) and (60) we find expressions of $\beta_{n}$ in terms of $x_{n}$ and of $\gamma_{n}$ in terms of $y_{n}$. Substituting them into (64), we find $y_{n+1}$ in terms of $x_{n}, \dot{x}_{n}$ and $y_{n}$. Substituting this expression into (62) we notice that we can express $y_{n}$ in terms of $x_{n}$ and $\dot{x}_{n}$. Expressions of $\beta_{n}$ and $\gamma_{n}$ in terms of $x_{n}$ and $y_{n}$ found earlier can also be substituted into equation (63), from where we get $\dot{y}_{n}$ in terms of $x_{n-1}$, $x_{n}$ and $y_{n}$. Substituting $x_{n-1}$ found at the beginning, we get equation for $\dot{y}_{n}$, $x_{n}$ and $y_{n}$. Hence, replacing $y_{n}$ by its expression in terms of $x_{n}$ and $\dot{x}_{n}$, we get the required second order differential equation for $x_{n}$.

\subsubsection{Differential equations using Toda systems}

In this subsection we assume that the parameter with respect to which we shall derive a differential equation is the parameter $t$ in the weight $w=$ 
$w_{0}(x) \exp (t x)$. In this case Theorem 4 is applicable. Throughout this subsection we assume the $t$-dependence of the polynomial $C$ in the Pearson equation $w^{\prime} / w=C / A$.

To derive differential equations we use the algorithm described at the beginning of the section. We have the following result.

Theorem 9 Let $w$ be a semi-classical weight such that $w^{\prime} / w=C / A$, with

$$
A(x)=x-\alpha, C(x)=c_{2} x^{2}+c_{1} x+c_{0}, \quad c_{2} \neq 0 .
$$

Let $\left\{P_{n}\right\}$ be the $S M O P$ with respect to $w$, satisfying the recurrence relation (6),

$$
P_{n+1}(x)=\left(x-\beta_{n}\right) P_{n}(x)-\gamma_{n} P_{n-1}(x), \quad n=0,1,2, \ldots
$$

Assuming a t-dependence in the weight of the form $w_{0}(x) \exp (t x)$ and, thus, the dependence on $t$ of the polynomial $C$, the function $x_{n}=x_{n}(t)$

$$
x_{n}=\frac{k_{1} c_{2}}{k_{2}\left(c_{1}+\alpha c_{2}\right)+\beta_{n} c_{2}}
$$

satisfies the following second order differential equation:

$\ddot{x}_{n}=\left(2-\frac{k_{2}^{2}}{2}\right) \frac{\dot{x}_{n}^{2}}{x_{n}}+\left(F_{1} x_{n}+F_{2}+F_{3} \frac{1}{x_{n}}\right) \dot{x}_{n}+F_{4} x_{n}^{3}+F_{5} x_{n}^{2}+F_{6} x_{n}+F_{7}+F_{8} \frac{1}{x_{n}}$,

where

$$
\begin{aligned}
F_{1}= & \frac{k_{2}^{2}\left(1-k_{2} \dot{c}_{1}\right)}{k_{1} c_{2}}+\frac{k_{2}^{3} c_{1} \dot{c}_{2}}{k_{1} c_{2}^{2}}, \\
F_{3}= & 2 k_{1}\left(1-\frac{1}{k_{2}^{2}}\right) \\
F_{2}= & \frac{2 \alpha}{k_{2}}-\frac{k_{2} c_{1}}{c_{2}}+\frac{c_{1}}{k_{2} c_{2}}-2 k_{2} \alpha, \\
F_{4}= & \frac{k_{1}^{2} c_{0}^{2}}{2 k_{2}^{2}}+\frac{k_{2}^{2}\left(2 k_{2} \dot{c}_{1}-k_{2}^{2} \dot{c}_{1}^{2}-1\right)}{2 k_{1}^{2} c_{2}^{2}}+\frac{k_{2}^{3}\left(k_{2} c_{1} \dot{c}_{1} \dot{c}_{2}-c_{1} \dot{c}_{2}\right)}{k_{1}^{2} c_{2}^{3}}-\frac{k_{2}^{4} c_{1}^{2} \dot{c}_{2}^{2}}{2 k_{1}^{2} c_{2}^{4}}, \\
F_{5}= & \frac{1}{k_{1} c_{2}}\left(2 k_{2} \alpha+\dot{c}_{0}+\alpha \dot{c}_{1}-2 k_{2}^{2} \alpha \dot{c}_{1}-k_{2} \ddot{c}_{1}\right)+\frac{k_{2}}{k_{1} c_{2}^{3}}\left(k_{2} c_{1}^{2} \dot{c}_{2}-2 c_{1} \dot{c}_{2}^{2}\right) \\
& +\frac{1}{k_{1} c_{2}^{2}}\left(k_{2} c_{1}-k_{2}^{2} c_{1} \dot{c}_{1}-\dot{c}_{2}-2 n \dot{c}_{2}-c_{0} \dot{c}_{2}+\left(2 k_{2}^{2}-1\right) \alpha c_{1} \dot{c}_{2}+2 k_{2} \dot{c}_{1} \dot{c}_{2}+k_{2} c_{1} \ddot{c}_{2}\right), \\
F_{6}= & \frac{1}{c_{2}}\left(2 k_{2} \dot{c}_{1}-c_{0}-3 \alpha c_{1}-2-2 n\right)-\frac{\dot{c}_{1}}{k_{2} c_{2}}-\frac{c_{1}}{2 c_{2}^{2}}\left(c_{1}+4 k_{2} \dot{c}_{2}\right)+\frac{c_{1} \dot{c}_{2}}{k_{2} c_{2}^{2}}-3 \alpha^{2},
\end{aligned}
$$




$$
\begin{aligned}
& F_{7}=\frac{4 k_{1} \alpha}{k_{2}}+\frac{2 k_{1} c_{2}}{k_{2} c_{2}}, \\
& F_{8}=-\frac{3 k_{1}^{2}}{2 k_{2}^{2}} .
\end{aligned}
$$

PROOF. The theorem is proved computationally using the algorithm for (32), (33), (34), (35) (with $t$ replaced by $\alpha$ in all four equations), (36) and (37).

Example 3 Let $A(x)=x-\alpha, C(x)=c_{2} x^{2}+c_{1} x+c_{0}$ with $c_{2}=2 b, c_{1}=t-2 \alpha b$, $c_{0}=\nu-\alpha t$. This case corresponds to the modified Laguerre weight considered in [20]. The weight function is given by

$$
w(x)=(x-\alpha)^{\nu} e^{b x^{2}+t x},
$$

where $b<0$. We notice that in this case and when $k_{2}=1$, the structure of the differential equation (66) resembles the alternative form of the fourth Painlevé equation (3). Indeed, if $\alpha=0$, then by the substitution $x_{n}(t)=$ $2 \sqrt{b} k_{1} / y(-t /(2 \sqrt{b}))$ we get that the function $y(z)$, where $z=-t /(2 \sqrt{b})$, satisfies the fourth Painlevé equation $P_{I V}\left(-1-2 n-\nu,-8 b^{2} k_{1}^{4} \nu^{2}\right)$. Note that in [20] only third order difference equation for the recurrence relation coefficients is given. However, as we see, in order to get the fourth Painlevé equation up to scaling transformation, one needs the extra condition $\alpha=0$. Moreover, if we take $k_{1}$ such that $4 b^{2} k_{1}^{4}=1$, then we have parameters of the fourth Painlevé equation satisfying condition (1). If additionally $b=-1$, then we have the case of the weight considered in [14] and the computations and the results agree by taking $k_{1}=-1 / \sqrt{2}$.

We can further study when equation (66) can be reduced to the fourth Painlevé equation up to a scaling transformation. By taking $x_{n}(t)=p_{1} / y\left(p_{2} t\right)$ we get that the function $y(z)$ with $z=p_{2}$ t satisfies $P_{I V}$ with $B=-p_{1}^{4}(-1-2 n+A)^{2} / 2$ when $k_{2}=1$ and $C(x)=-x^{2} /\left(2 p_{2}^{2}\right)+x t+\sqrt{-2 B} / p_{1}^{2}, A(x)=x$, which esentially gives the case considered at the beginning of this example.

\subsubsection{Differential equations using Toda-type system}

In this subsection we assume that the parameter with respect to which we shall derive a differential equation is the root of $A(x)$, that is, $A(x)=x-t$. We also assume the $t$-dependence of the polynomial $C$ in the Pearson equation $w^{\prime} / w=C / A$. Now, Theorem 5 is applicable. We have the following result.

Theorem 10 For the SMOP with respect to $w$ such that $w^{\prime} / w=C / A$ with $A(x)=x-t, C(x)=c_{2} x^{2}+c_{1} x+c_{0}$, the recurrence relation coefficients of 
(6) satisfy the following Toda-type system:

$$
\left\{\begin{array}{l}
\dot{\gamma}_{n}=c_{2} \gamma_{n}\left(\beta_{n}-\beta_{n-1}\right), \\
\dot{\beta}_{n}=1+c_{2}\left(\gamma_{n+1}-\gamma_{n}\right) .
\end{array}\right.
$$

PROOF. We have

$$
\Theta_{n}(x)=-\left(c_{1}+c_{2} x+c_{2} \beta_{n+1}\right) \gamma_{n+1}
$$

and

$$
l_{n}(x)=1+n+\left(c_{0}+c_{1} x+c_{2} x^{2}+2 c+2 \gamma_{n+1}\right) / 2 .
$$

Substituting these expresions to (38) and (39) with $m=1$ we get the desired result.

To derive differential equations we use the algorithm described at the beginning of the section. We have the following result.

Theorem 11 Let $w$ be a semi-classical weight such that $w^{\prime} / w=C / A$, with

$$
A(x)=x-t, C(x)=c_{2} x^{2}+c_{1} x+c_{0}, \quad c_{2} \neq 0 .
$$

Let $\left\{P_{n}\right\}$ be the SMOP with respect to $w$, satisfying the recurrence relation (6),

$$
P_{n+1}(x)=\left(x-\beta_{n}\right) P_{n}(x)-\gamma_{n} P_{n-1}(x), \quad n=0,1,2, \ldots .
$$

The function $x_{n}=x_{n}(t)$

$$
x_{n}=\frac{k_{1} c_{2}}{k_{2}\left(c_{1}+c_{2} t\right)+\beta_{n} c_{2}}
$$

satisfies the following second order differential equation:

$\ddot{x}_{n}=\left(2-\frac{k_{2}^{2}}{2}\right) \frac{\dot{x}_{n}^{2}}{x_{n}}+\left(G_{1} x_{n}+G_{2}+G_{3} \frac{1}{x_{n}}\right) \dot{x}_{n}+G_{4} x_{n}^{3}+G_{5} x_{n}^{2}+G_{6} x_{n}+G_{7}+G_{8} \frac{1}{x_{n}}$,

where

$$
\begin{aligned}
& G_{1}=\frac{k_{2}^{3} c_{1} \dot{c}_{2}}{k_{1} c_{2}^{2}}-\frac{k_{2}^{3} \dot{c}_{1}}{k_{1} c_{2}}-\frac{k_{2}^{3}}{k_{1}}, \\
& G_{2}=\frac{c_{1}+2 c_{2} t}{k_{2}}-k_{2}\left(c_{1}-2 c_{2} t\right)+\frac{\dot{c}_{2}}{c_{2}} \\
& G_{3}=2 k_{1} c_{2}\left(1-\frac{1}{k_{2}^{2}}\right), \\
& G_{4}=-\frac{k_{2}^{4}}{2 k_{1}^{2}}+\frac{k_{1}^{2} c_{0}^{2} c_{2}^{2}}{2 k_{2}^{2}}-\frac{k_{2}^{4} \dot{c}_{1}}{k_{1}^{2} c_{2}}+\frac{k_{2}^{4} c_{1} \dot{c}_{1} \dot{c}_{2}}{k_{1}^{2} c_{2}^{3}}-\frac{k_{2}^{4} c_{1}^{2} \dot{c}_{2}^{2}}{2 k_{1}^{2} c_{2}^{4}}+\frac{k_{2}^{4}\left(2 c_{1} \dot{c}_{2}-\dot{c}_{1}^{2}\right)}{2 k_{1}^{2} c_{2}^{2}},
\end{aligned}
$$




$$
\begin{aligned}
G_{5}= & \frac{1}{k_{1}}\left(c_{1}-k_{2}^{2} c_{1}+2 c_{2} t-2 k_{2}^{2} c_{2} t+\dot{c}_{0}+\dot{c}_{1} t-2 k_{2}^{2} \dot{c}_{1} t\right) \\
& \quad+\frac{1}{k_{1} c_{2}}\left(k_{2} \dot{c}_{2}-k_{2}^{2} c_{1} \dot{c}_{1}-2 n \dot{c}_{2}-c_{0} \dot{c}_{2}-c_{1} \dot{c}_{2} t+2 k_{2}^{2} c_{1} \dot{c}_{2} t-k_{2} \ddot{c}_{1}\right) \\
& \quad+\frac{1}{k_{1} c_{2}^{2}}\left(k_{2}^{2} c_{1}^{2} \dot{c}_{2}+3 k_{2} \dot{c}_{1} \dot{c}_{2}+k_{2} c_{1} \ddot{c}_{2}\right)-\frac{3 k_{2} c_{1} \dot{c}_{2}^{2}}{k_{1} c_{2}^{3}}, \\
G_{6}= & \frac{c_{1} \dot{c}_{2}}{k_{2} c_{2}}-\frac{2 k_{2} c_{1} \dot{c}_{2}}{c_{2}}-\frac{\dot{c}_{1}+2 c_{2}}{k_{2}}-\frac{c_{1}^{2}}{2}+2 k_{2} \dot{c}_{1}-3 c_{1} c_{2} t-c_{2}\left(2 n-2 k_{2}+c_{0}+3 c_{2} t^{2}\right), \\
G_{7}= & \frac{2 k_{1} c_{1} c_{2}+4 k_{1} c_{2}^{2} t}{k_{2}}, \\
G_{8}= & -\frac{3 k_{1}^{2} c_{2}^{2}}{2 k_{2}^{2}} .
\end{aligned}
$$

PROOF. The theorem is proved computationally using the algorithm for (32), (33), (34), (35), (67).

Example 4 Let $A(x)=x-t, C(c)=c_{2} x^{2}+c_{1} x+c_{0}$ with $c_{2}=2 b, c_{1}=a-2 b t$, $c_{0}=\nu-$ at. This case corresponds to the modified Laguerre weight considered in [20]. The weight function is given by

$$
w(x)=(x-t)^{\nu} e^{b x^{2}+a x}
$$

where $b<0, a<0$. We notice that in this case and when $k_{2}=1$, the structure of the differential equation (69) resembles the alternative form of the fourth Painlevé equation (3). Indeed, if $a=0$, then by the substitution $x_{n}(t)=-2 \sqrt{b} k_{1} / y(\sqrt{b} t)$ we get that the function $y(z)$, where $z=\sqrt{b} t$, satisfies the fourth Painlevé equation $P_{I V}\left(-1-2 n-\nu,-8 b^{2} k_{1}^{4} \nu^{2}\right)$. Note that in [20] only difference equation for the recurrence relation coefficients is given and it is conjectured that if one obtains the differential equation with respect to the parameter, then one gets one of the Painlevé equations. However, as we see, in order to get the fourth Painlevé equation up to scaling transformation, one needs the extra condition $a=0$. Moreover, if we take $k_{1}$ such that $4 b^{2} k_{1}^{4}=1$, then we have parameters of the fourth Painleve equation satisfying condition (1). If additionally $b=-1$, then we have the case of the weight considered in [1] $\left(w(x)=|x-t|^{\nu} e^{-x^{2}}\right)$, and by a scaling we get that the recurrence relation coefficients are related to $P_{I V}\left(1+2 n+\nu,-2 \nu^{2}\right)$, which agrees with the result in [7].

We can further study when equation (69) can be reduced to the fourth Painlevé equation up to a scaling transformation. By taking $x_{n}(t)=p_{1} / y\left(p_{2} t\right)$ we get that the function $y(z)$ with $z=p_{2}$ t satisfies $P_{I V}\left(1-2 n-\nu,-8 \nu^{2} k_{1}^{4} p_{2}^{4}\right)$ when $k_{2}=1$ and $C(x)=-2 p_{2}^{2} x^{2}+2 p_{2}^{2} t x+\nu$, which gives the case considered at the beginning of this example with $b=-p_{2}^{2}, a=0$. 


\section{Discussion}

In this paper we derived general differential equations for the recurrence relation coefficients of the semi-classical orthogonal polynomials of class one with an additional assumption that the degree of $A$ in (14) or (15) is at most one. In particular cases we showed a connection to the second and the fourth Painlevé equations. Moreover, we considered the modified Laguerre weight (70) (see [20]) and deduced differential equations with respect to two different parameters.

In general terms, our approach uses a method similar as in [14]. Other methods are available in the extensive literature on Painlevé equations and semiclassical orthogonal polynomials. For instance, some differential equations for $\beta_{n}$ deduced in Theorem 6 can also be obtained using pairs of Backlund transformations as in [11].

There are several open problems to be emphasized. Firstly, by considering different combinations on roots of $A$, to show a connection of the recurrence relation coefficients to other discrete and differential Painlevé equations. Another open problem is to deduce differential equations in larger classes than the semi-classical one, for instance, the Laguerre-Hahn class [17]. Here, in the account of the results of [13], we conjecture that the structure of these equations will be similar to the case considered in this paper, and for some examples of Laguerre-Hahn polynomials, for instance in the case of the associated polynomials, there will also be a connection to the Painlevé equations. Finally, it would also be interesting to systematically study the general equations to find all the cases when we have the second and the fourth Painlevé equations, not necessarily up to scaling transformations.

\section{Acknowledgements}

The authors are very grateful to the anonymous referee for her/his valuable suggestions and comments, which improved the paper significantly.

The work of MNR was partially supported by the Centre for Mathematics of the University of Coimbra - UID/MAT/00324/2013, funded by the Portuguese Government through FCT/MCTES and co-funded by the European Regional Development Fund through the Partnership Agreement PT2020.

GF acknowledges the support of the Alexander von Humboldt foundation and the hospitality of KU Eichstätt-Ingolstadt. 


\section{References}

[1] J. Alaya and P. Maroni, Symmetric Laguerre-Hahn forms of class $s=1$, Integral Transforms Spec. Funct. 4 (1996), 301-320.

[2] E. Basor, Y. Chen and T. Ehrhardt, Painlevé $V$ and time-dependent Jacobi polynomials, J. Phys. A 43 (2010), 015204 (25 pp.).

[3] P. Bleher, A. Its, Semiclassical asymptotics of orthogonal plynomials, RiemannHilbert problem, and universality in the matrix model, Ann. Math. 150 (1999), $185-266$.

[4] A. Branquinho, A. Paiva and M.N. Rebocho, Sylvester equations for LaguerreHahn orthogonal polynomials on the real line, Appl. Math. Comput. 219 (2013), 9118-9131.

[5] C. Brezinski, A direct proof of the Christoffel-Darboux identity and its equivalence to the recurrence relationship, J. Comput. Appl. Math. 32 (1-2) (1990), 1-75.

[6] Y. Chen and D. Dai, Painlevé $V$ and a Pollaczek-Jacobi type orthogonal polynomials, J. Approx. Theory 162 (2010), 2149-2167.

[7] Y. Chen and M.V. Feigin, Painlevé IV and degenerate Gaussian unitary ensembles, J. Phys. A: Math. Gen. 39 (2006), 12381-12393.

[8] Y. Chen and A. Its, Painlevé III and a singular linear statistics in Hermitian random matrix ensembles. I, J. Approx. Theory 162 (2010), 270-297.

[9] P.A. Clarkson, Painlevé transcendents, in: NIST handbook of mathematical functions, 723-740, U.S. Dept. Commerce, Washington, DC, 2010.

[10] P.A. Clarkson and K. Jordaan, The relationship between semiclassical Laguerre polynomials and the fourth Painlevé equation, Constr. Approx. 39 (2014), 223254.

[11] P.A. Clarkson, A.F. Loureiro, W. Van Assche, Unique positive solution for alternative d-PI, J. Difference Equ. Appl. 22 (2016) 656-675.

[12] M.V. Fedoryuk, Isomonodromy deformations of equations with irregular singularities, Math. Sb. 181 (1990); Math. USSR Sb. 71 (1992), 463-479.

[13] G. Filipuk and M. N. Rebocho, Discrete Painlevé equations for recurrence coefficients of Laguerre-Hahn orthogonal polynomials of class one, Integral Transforms Spec. Funct. 27 (2016) 548-565.

[14] G. Filipuk, W. Van Assche and L. Zhang, The recurrence coefficients of semiclassical Laguerre polynomials and the fourth Painlevé equation, J. Phys. A: Math. Theor. 45 (2012), 205201 (13pp.).

[15] P. Forrester and C. Ormerod, Differential equations for deformed Laguerre polynomials, J. Approx. Theory 162 (2010), 653-677. 
[16] A.P. Magnus, Painlevé-type differential equations for the recurrence coefficients of semi-classical orthogonal polynomials, J. Comput. Appl. Math. 57 (1995), $215-237$.

[17] F. Marcellán and E. Prianes, Orthogonal polynomials and Stieltjes functions: the Laguerre-Hahn case, Rend. Mat. Appl. (1996), 117-141.

[18] P. Maroni, Une théorie algébrique des polynômes orthogonaux. Application aux polynômes orthogonaux semi-classiques, in: Orthogonal polynomials and their applications (Erice 1990), 95-130, IMACS Ann. Comput. Appl. Math. 9, Baltzer, Basel, 1991.

[19] J. Moser, Finately many mass points on the line under the influence of an exponential potential - an integrable system, in: Dynamical systems, Theory and Applications, J. Moser (Editor), pp. 469-497, Lecture Notes in Physics, vol. 38, Springer-Verlag, Berlin, 1975.

[20] P. E. Spicer and F. W. Nijhoff, Semi-classical Laguerre polynomials and a thirdorder discrete integrable equation, J. Phys. A: Math. Theor. 42 (2009), 454019 (9pp.).

[21] G. Szegő, Orthogonal Polynomials, Amer. Math. Soc. Colloq. Publ., vol. 23, Amer. Math. Soc. Providence Rhode Island, 1975 (fourth edition).

[22] W. Van Assche, Discrete Painlevé equations for recurrence coefficients of orthogonal polynomials, In: Elaydi S, Cushing J, Lasser R, Ruffing A, Papageorgiou V, Van Assche W, editors. Difference equations, special functions and orthogonal polynomials. Hackensack, NJ: World Scientific; 2007. p. 687-725. 